\title{
Interfacing carbon nanotubes (CNT) with plants: enhancement of growth, water and ionic nutrient uptake in maize (Zea mays) and implications for nanoagriculture
}

\author{
D. K. Tiwari $\cdot$ N. Dasgupta-Schubert $\cdot$ \\ L. M. Villaseñor Cendejas $\cdot$ J. Villegas $\cdot$ \\ L. Carreto Montoya $\cdot$ S. E. Borjas García
}

Received: 15 April 2013/Accepted: 15 May 2013/Published online: 2 June 2013

(C) The Author(s) 2013. This article is published with open access at Springerlink.com

\begin{abstract}
The application of nano-biotechnology to cropscience/agriculture ('nanoagriculture') is a recent development. While carbon nanotubes (CNTs) have been shown to dramatically improve germination of some comestible plants, deficiencies in consistency of behavior and reproducibility arise, partially from the variability of the CNTs used. In this work, factory-synthesized multi-walled-CNTs (MWCNTs) of quality-controlled specifications were seen to enhance the germinative growth of maize seedlings at low concentrations but depress it at higher concentrations. Growth enhancement principally arose through improved water delivery by the MWCNT. Polarized EDXRF spectrometry showed that MWCNTs affect mineral nutrient supply to the seedling through the action of the mutually opposing forces of inflow with water and retention in the medium by the ion-CNT transient-dipole interaction. The effect varied with ion type and MWCNT concentration. The differences of the Fe tissue concentrations when relatively high equimolar $\mathrm{Fe}^{2+}$ or $\mathrm{Fe}^{3+}$ was introduced, implied that the ion-CNT interaction might induce redox changes to the ion. The tissue $\mathrm{Ca}^{2+}$ concentration manifested as the antipode of the $\mathrm{Fe}^{2+}$ concentration indicating a possible cationic exchange in the cell wall matrix. SEM images showed that MWCNTs perforated the black-layer seed-coat that could explain the enhanced water delivery.
\end{abstract}

D. K. Tiwari · L. M. Villaseñor Cendejas · S. E. Borjas García Radiation Laboratory, Institute of Physics and Mathematics (IFM), University of Michoacan (UMSNH), Cd. Universitaria, C.P. 58060 Morelia, Michoacan, Mexico

N. Dasgupta-Schubert $(\varangle) \cdot$ J. Villegas $\cdot$ L. Carreto Montoya Phytotechnology Laboratory, Institute of Chemical Biology (IIQB), University of Michoacan (UMSNH), Cd. Universitaria, C.P. 58060 Morelia, Michoacan, Mexico

e-mail: nita@ifm.umich.mx
The absence of perforations with the introduction of $\mathrm{FeCl}_{2} /$ $\mathrm{FeCl}_{3}$ reinforces the idea of the modification of MWCNT functionality by the ion-CNT interaction. Overall, in normal media, low dose MWCNTs were seen to be beneficial, improving water absorption, plant biomass and the concentrations of the essential $\mathrm{Ca}, \mathrm{Fe}$ nutrients, opening a potential for possible future commercial agricultural applications.

Keywords MWCNT $\cdot$ Maize $\cdot$ Germination $\cdot$ Ion · Nano-biotechnology $\cdot$ Nanoagriculture

\section{Introduction}

During the last decade, noteworthy advances have been reported in the study of the fundamental characteristics of nanomaterials and their utilization for a multitude of applications. In medicine and biology, nanomaterials have been investigated for diverse end-uses such as gene and drug delivery, bio-sensing and diagnostic and tissue engineering (Panyam and Labhatsevar 2003; Merkoçi 2009; Tiwari and Tiwari 2013; Lee et al. 2010). Since their discovery (Ijima 1991), carbon nanotubes (CNT) amongst all nano materials, have attracted a major share of the interest because of their unique mechanical, electrical, thermal and chemical properties (Dresselhaus et al. 2004). Most studies of CNTs in the biosciences have focused on their influence on animal and human cells (Donaldson et al. 2006; Martinelli et al. 2012). Relatively scant attention has been paid to the effect of CNTs on plant cells and the way they might influence the physiology and the development of plants. Yet plants are fundamental to higher life on earth-they are the geo-physico-chemical transducers that produce food and oxygen that sustain life in the biosphere. They are also 
end-receivers of environmental contaminants including the CNT-based ones. Thus the plant-CNT interaction needs to be thoroughly investigated from the cellular to the organismic level to understand its multifaceted complexity. This knowledge will also serve to develop the technology of 'nanoagriculture' - a nascent area of nano-biotechnology that holds promise for growth acceleration and higher biomass yields of crop plants (Srinivasan and Saraswathi 2010).

At the cellular level there has been substantial interest in the possibility of CNTs penetrating the plant cell wall to work as a smart delivery system of chemicals to the cell. The ability of single-walled-CNTs (SWCNTs) to penetrate the cell wall and membrane of tobacco cells (Liu et al. 2009) and of cup-stacked cellulase impregnated CNTs to penetrate the cell wall and transport intracellularly through cellulase-induced nanoholes (Serag et al. 2012), have been demonstrated. At the whole plant level, a number of researchers have reported the dramatic effects on seed germination and plant growth by multi-walled CNTs (MWCNTs). MWCNTs have been shown to penetrate the seed-coat and stimulate the growth of tomato seeds (Khodakovskaya et al. 2009; Villagarcia et al. 2012) as well as the growth of mustard seeds (Mondal et al. 2011), while water soluble MWCNTs (wsMWCNT) have been shown to exhibit the same dramatic improvement of the growth of gram plants (Tripathi et al. 2011). More efficient water uptake induced by the CNT has been implied as the growth stimulator (Khodakovskaya et al. 2009; Villagarcia et al. 2012; Mondal et al. 2011; Tripathi et al. 2011). On the other hand there exist contradictory reports that show the toxicity of CNTs to the growth of a number of different plants (Begum et al. 2012; Lin and Xing 2007). MWCNT reduced the root fresh weights (FW) of rice and cucumber seedlings while the root lengths (RL) varied in a nonorderly manner with MWCNT concentration (rice) or decreased (cucumber) (Begum et al. 2012); the germination rate of maize and rye grass decreased with $2,000 \mathrm{mg} / \mathrm{l}$ of MWCNT but their RLs increased (Lin and Xing 2007); MWCNT increased the FW and RLs of wheat seedlings but had no effect on seed germination and shoot lengths (SL) (Wang et al. 2012).

The contradictory observations are a result of the variability between the experimental entities employed in the studies. The effect of the MWCNT will principally vary with the type of plant studied, the type of growth medium and ambient growth conditions, the type of MWCNT (pristine or functioanlised), its concentration, and the ultrasonication and associated techniques used to obtain the aqueous dispersion of the MWCNT. The dependence of seedling growth on the first three factors is well known in plant physiology. An assortment of media have been used in the aforesaid works-wet filter paper (Mondal et al.
2011; Lin and Xing 2007; Wang et al. 2012), agar with MS nutrients (Khodakovskaya et al. 2009; Villagarcia et al. 2012) and hydroponics with Hoagland's nutrients (Begum et al. 2012) or without (Tripathi et al. 2011). Pristine MWCNTs are hydrophobic and form aggregates in aqueous media that reduce their aspect ratio and hence their effectiveness. To disperse their CNTs the researchers in the aforesaid studies had functionalised their CNTs with different hydrophilic groups (Khodakovskaya et al. 2009; Villagarcia et al. 2012; Mondal et al. 2011; Tripathi et al. 2011) or had used a surfactant (PEG) (Villagarcia et al. 2012), and had used different protocols for ultrasonication (Khodakovskaya et al. 2009; Villagarcia et al. 2012; Mondal et al. 2011; Tripathi et al. 2011; Lin and Xing 2007; Wang et al. 2012). Functionalization and micellar cage formation by a surfactant (Vaisman et al. 2006) alter the surface chemistry of the CNT, which changes the way it interacts with the plant (Villagarcia et al. 2012). Furthermore, different durations or intensities of ultrasonication will change the degree of dispersion of the CNT, introducing its component to the variability. Too vigorous an ultrasonication has been supposed damage the CNT (Vaisman et al. 2006). In yet another variation, ultrasonication had been replaced by bead milling (Begum et al. 2012). Pristine MWCNTs where used were pure, but were individualistic in type because they had been fabricated in the researchers' own laboratories with different techniques, resulting in MWCNTs with different morphologies or remnant functional groups. Thus the substantial variability in the characteristics of the CNTs used is also the reason for the variable observations.

To define the basic response of the given plant to the MWCNT unambiguously, a baseline study that can be easily replicated across independent laboratories becomes necessary. Such a study would incorporate standard components of controlled specifications that are universally available, utilize minimal processing, and for the plant growth, employ a simple well-established protocol. Such a baseline could also serve as reference to more elaborate studies involving different processing techniques, growth protocols and other features. Also, if CNT-driven nanoagriculture is to be developed as a large-scale commercial venture, consistency and reproducibility of results acquire added importance. The general goal of the present work is a baseline-type study with add-ons as described further below.

We report the study of maize (Zea mays) seedlings grown in nutrient agar gel under controlled ambient conditions with the gel being treated by different concentrations of pristine MWCNT non-ultrasonically distributed within it. The MWCNT were obtained from an established chemicals company and possessed reliable, quality-controlled specifications. Maize is one of the species 
recommended by the USEPA (US Environmental Protection Agency 1996) for the determination of the ecological effects of toxic substances. Moreover, it is an important food and agro-industrial crop such that an enhancement of its growth rate and biomass production (if CNTs do indeed promote these) would be highly beneficial. Few studies of CNT affected seed germination have been conducted on maize. The objective of the present study was to observe the effect of the MWCNT on the indices of growth and water absorption of the whole seedling as well as its morphological parts and to determine the mineral nutrient concentrations in the seedling. Seedling growth in the initial phases of seed germination is principally driven by water imbibition (MacDonald 2007 and Private Communication Feb. 2013). If the mechanism of the CNT-seed interaction is through the facilitation of water uptake, then this is also expected to enhance mineral nutrient uptake and affect growth. However, given the fact that CNTs purportedly display a marked interaction with ions and polar molecules (Miskovic 2008; Frolov et al. 2012), it would be interesting to see how enhanced water delivery versus ionCNT interactions play out as far as seedling nutrient uptake is concerned. The mechanism of ionic interactions with the CNT surface (Miskovic 2008) implies that redox type changes of the nutrient ion of a given oxidation state might take place with the MWCNTs in the medium. This is explored in the later part of this work with the introduction of the important mineral nutrient iron as $\mathrm{Fe}(\mathrm{II})$ and $\mathrm{Fe}(\mathrm{III})$ chloride in agarose gel. Agarose was chosen because it retains the gel platform of the previous study but is practically nutrient free so that the effects on iron will not be obscured by interference by other ions or polar species. The initiation of germination is not affected by the lack of substrate nutrients because it is driven by the stored food in the seed endoderm/cotyledon (MacDonald 2007 and Private Communication Feb 2013). The growth experiments were terminated after 7 days of growth because up till $\sim 7$ days, the phases I-III of germination predominate where as stated before, water imbibition is of paramount importance.

\section{Experimental section}

Materials

MWCNTs were purchased from Sigma-Aldrich ${ }^{\circledR}$ (St. Louis, MO, USA), purity $>95 \%$, OD 6-9 nm, L $5 \mu \mathrm{m}$. Bacteriological agar (BA) (Bioxon ${ }^{\circledR}$ ) was purchased from Becton-Dickinson (BD de Mexico, Mexico City, Mexico) and Agarose from Sigma-Aldrich ${ }^{\circledR}$ (A9539). BA contains a certain level of mineral nutrients as ionic or polar species (Difco $^{\mathrm{TM}}$ and $\mathrm{BBL}^{\mathrm{TM}}$ manual 2009) but their levels in agarose are negligible. The reagents $\mathrm{FeCl}_{2} \cdot 4 \mathrm{H} 2 \mathrm{O}(>99 \%$ pure) and $\mathrm{FeCl}_{3} \cdot 6 \mathrm{H} 2 \mathrm{O}$ ( $\geq 97 \%$ pure) were purchased from Sigma-Aldrich ${ }^{\circledR}$ and Mallinckrodt Inc. (Phillipsburg, NJ, USA), respectively. Maize (sweet corn) seeds were purchased from a local seed supply company (Hortaflor, Rancho Los Molinos SA de CV, Mexico).

\section{Substrate preparation and seedling growth}

The first experimental set (EXP-1) consisted of maize seedlings grown in BA gel medium spiked with different concentrations of MWCNT.

The BA powder was weighed and mixed with de-ionised (DI) water to obtain a concentration of $15 \mathrm{~g} / \mathrm{l}$ and then autoclaved at $120{ }^{\circ} \mathrm{C}$ for $20 \mathrm{~min}$. Appropriate masses of MWCNT were weighed out and mixed with aliquot volumes of the sterile BA solution by mechanical stirring for $\sim 10 \mathrm{~min}$ in order to yield unvaried distributions of MWCNT in the gel of the different concentrations listed in Table 1. The BA solutions containing the different concentrations of the MWCNT were then poured into replicate sterile petri dishes to set as the gel substrate for seed growth.

Maize seeds were cleaned by magnetic stirring in a $70 \%$ ethanol solution for $2 \mathrm{~min}$ and washed with DI water. They were then surface sterilized in a $1 \% \mathrm{v} / \mathrm{v}$ solution of sodium hypochlorite by gentle stirring for $10 \mathrm{~min}$, then washed several times with DI water. The seeds were blotted dry in sterile paper towels and implanted into the set gel substrates described previously. The petri dishes were sealed with tape and appropriately labeled. These operations were done in the laminar flow-hood. The petri dishes were then transferred to a climate-controlled chamber (temperature $23-25{ }^{\circ} \mathrm{C}$, relative humidity (RH) $\sim 71.5 \%$, photoperiod $16 \mathrm{~h}$ light- $8 \mathrm{~h}$ dark) and the seeds allowed to germinate and grow for 7 days. The total number of seedlings was 108 with each MWCNT case listed in Table 1 containing 18 seedlings.

The second experimental set (EXP-2) was identical to the first except that only the Control and the MWCNT concentration of $20 \mathrm{mg} / \mathrm{l}$ were used and a subset of seedlings was harvested periodically for the time-series analysis

Table 1 Concentrations of the MWCNT ([MWCNT]) prepared in the bacteriological agar (BA) medium

\begin{tabular}{|c|c|}
\hline $\begin{array}{l}\text { Sample } \\
\text { name }\end{array}$ & $\begin{array}{l}\text { Carbon nanotube } \\
\text { concentration }(\mathrm{mg} / \mathrm{l})\end{array}$ \\
\hline 0 & 0 \\
\hline 5 & 5 \\
\hline 10 & 10 \\
\hline 20 & 20 \\
\hline 40 & 40 \\
\hline 60 & 60 \\
\hline
\end{tabular}


Table 2 Concentrations of $\mathrm{Fe}(\mathrm{II})$ and $\mathrm{Fe}(\mathrm{III})$ introduced as their chlorides and the concentration of the MWCNT in the agarose medium

\begin{tabular}{ll}
\hline Sample name & Additive concentration \\
\hline A0 & - \\
A1 & MWCNTs $(20 \mathrm{mg} / \mathrm{l})$ \\
A2 & $\mathrm{Fe}(\mathrm{II}) 3 \times 10^{-4} \mathrm{M}$ \\
A3 & $\mathrm{Fe}(\mathrm{II}) 3 \times 10^{-4} \mathrm{M}+$ MWCNTs $(20 \mathrm{mg} / \mathrm{l})$ \\
A4 & $\mathrm{Fe}(\mathrm{III}) 3 \times 10^{-4} \mathrm{M}$ \\
A5 & $\mathrm{Fe}(\mathrm{III}) 3 \times 10^{-4} \mathrm{M}+$ MWCNTs $(20 \mathrm{mg} / \mathrm{l})$ \\
\hline
\end{tabular}

of the growth. The progress of seedling development in the two experimental sets was monitored photographically.

The third experimental set (EXP-3) consisted of maize seedlings grown in agarose gel medium spiked with $\mathrm{Fe}(\mathrm{II})$ and $\mathrm{Fe}$ (III) chloride with or without the simultaneous presence of MWCNT at the concentration of $20 \mathrm{mg} / \mathrm{l}$.

Agarose powder was weighed and mixed in DI water, so as to correspond to a concentration of $8 \mathrm{~g} / \mathrm{l}$. The agarose was dissolved by heating the aqueous mixture at $<95{ }^{\circ} \mathrm{C}$ with constant magnetic stirring for about $2.5 \mathrm{~h}$. Upon complete dissolution, different aliquot volumes of the clear agarose solution were measured out and the appropriate masses of $\mathrm{Fe}$ (II) and $\mathrm{Fe}(\mathrm{III})$ chloride as well as the appropriate mass of the MWCNT were mixed in by magnetic stirring for $\sim 10 \mathrm{~min}$ to yield the concentrations as presented in Table 2 . The Fe concentration of $3 \times 10^{-4} \mathrm{M}$ $(16.8 \mathrm{mg} / \mathrm{l})$ approximated the MWCNT concentration of $20 \mathrm{mg} / \mathrm{l}$. The agarose solutions containing these different species were then poured into replicate sterile petri dishes to set as the gel substrate for seed growth. The protocol for seed implantation was the same as in the first experimental set. The total number of seedlings in this set was 243 with each of the cases listed in Table 2 containing 40-41 seedlings. The growth for all seedlings was carried out in the dark for 7 days at the ambient conditions of $\sim 23{ }^{\circ} \mathrm{C}$ and a $\mathrm{RH}$ of $\sim 55 \%$. Growth in the dark was necessary to prevent the photo-oxidation of $\mathrm{Fe}(\mathrm{II})$ (Brateman et al. 1984). Like many cultivated species, maize seeds are able to germinate equally well in light or dark.

\section{Morphological measurements and Microscopy}

Upon harvesting, the seedlings were gently rinsed with DI water, blotted dry with paper towels and their FW, RL and SL measured. The roots and shoots were then cut and the FWs of these parts as well as of the seed body were measured. A few seed bodies from the third experimental set were analyzed by Scanning Electron Microscopy (SEM). The seed parts were then oven dried at $60{ }^{\circ} \mathrm{C}$ for $72 \mathrm{~h}$. The dry weights (DW) of these parts were then measured.
The SEM analysis was performed for the surface topography of the pericarp/seed-coat near the black-layer of the maize seed where the radicle emerges. The seed body was copper coated by vacuum deposition and analyzed in the field emission SEM (JSM 7401F, JEOL Ltd. Tokyo, Japan) at $15 \mathrm{kV}$ with a working distance of 28-30 mm.

Elemental analysis of the seedling tissue by polarized energy dispersive X-ray fluorescence analysis (pEDXRF)

pEDXRF is an advancement in EDXRF instrumentation first proposed in the mid-1990s (Heckel et al. 1992) with commercial instruments sold since the early 2000s. The operating principle is based on the cancellation of the Bremmstrahlung background by the double polarization of the initial X-ray beam. Consequently the analytical sensitivity markedly improves, permitting trace level analysis. We have shown (Alvarado et al. 2013 in press) that samples of masses lower than the recommended mass for ideal analysis (as frequently occur in the biosciences), can be analyzed for relative elemental concentrations with sufficient accuracy. The detectable elemental range is from $\mathrm{Na}$ to $U$. The advantages of pEDXRF lie in its non-destructive, multielemental and rapid trace analysis capabilities.

Dry plant parts were ball milled (model MM-400, Retsch GmbH, Germany) at $25 \mathrm{~Hz}$ for 2 min till the entire mass appeared as a fine powder. The elemental analysis of the powders was carried out by the pEDXRF spectrometer SpectroXepos III (Spectro Analytical Instruments GmbH, Kleve, Germany) using the TurboQuant ${ }^{\mathrm{TM}}$ analytical routine. It is important to remember that for the low sample dry mass yields as in the present work, the quantity of interest is the relative magnitude of the element's concentration corresponding to the different treatments.

Data analysis

Averages and standard errors were computed over 3 sets of replicates for each case listed in the Tables 1 and 2 with the number of seedlings for each set as cited previously. Tests of statistical differences were carried out by the Student's $t$ test at $95 \%$ confidence level, except where stated differently. The softwares (Origin Lab) ORIGIN 6 and SIGMAPLOT 10 were used for data reduction and analysis.

\section{Results}

Figure 1a-d show the various indices studied in this work as they vary with the MWCNT concentration ([MWCNT]) in the BA medium for the fixed period of germination of 
7 days (EXP-1). The \% water was calculated as: $\{(\mathrm{FW}-\mathrm{DW}) /$ FW $\} \times 100$. The MWCNT increased the water content of the root but less unequivocally for the whole seedling (Fig. 1a). The sudden increase at low [MWCNT] continued in a gradual manner or attained a near constancy at the higher [MWCNT]. For the shoot however, after a spurt in the \%water at the [MWCNT] of $10 \mathrm{mg} / \mathrm{l}$, the water content decreased gradually. The trace for the seedling follows the combined response of the root and shoot. The FWs (Fig. 1c) follow the trends of the water contents, which is reasonable due to $\mathrm{FW}$ being largely composed of tissue water. The DWs (Fig. 1d) for each morphological part are not much different at the low [MWCNT] but show a sudden spurt at $20 \mathrm{mg} / 1$ [MWCNT] particularly for the root, while slowly declining at the higher [MWCNT]. Figure 1b shows that the RLs increased markedly with root \%water;
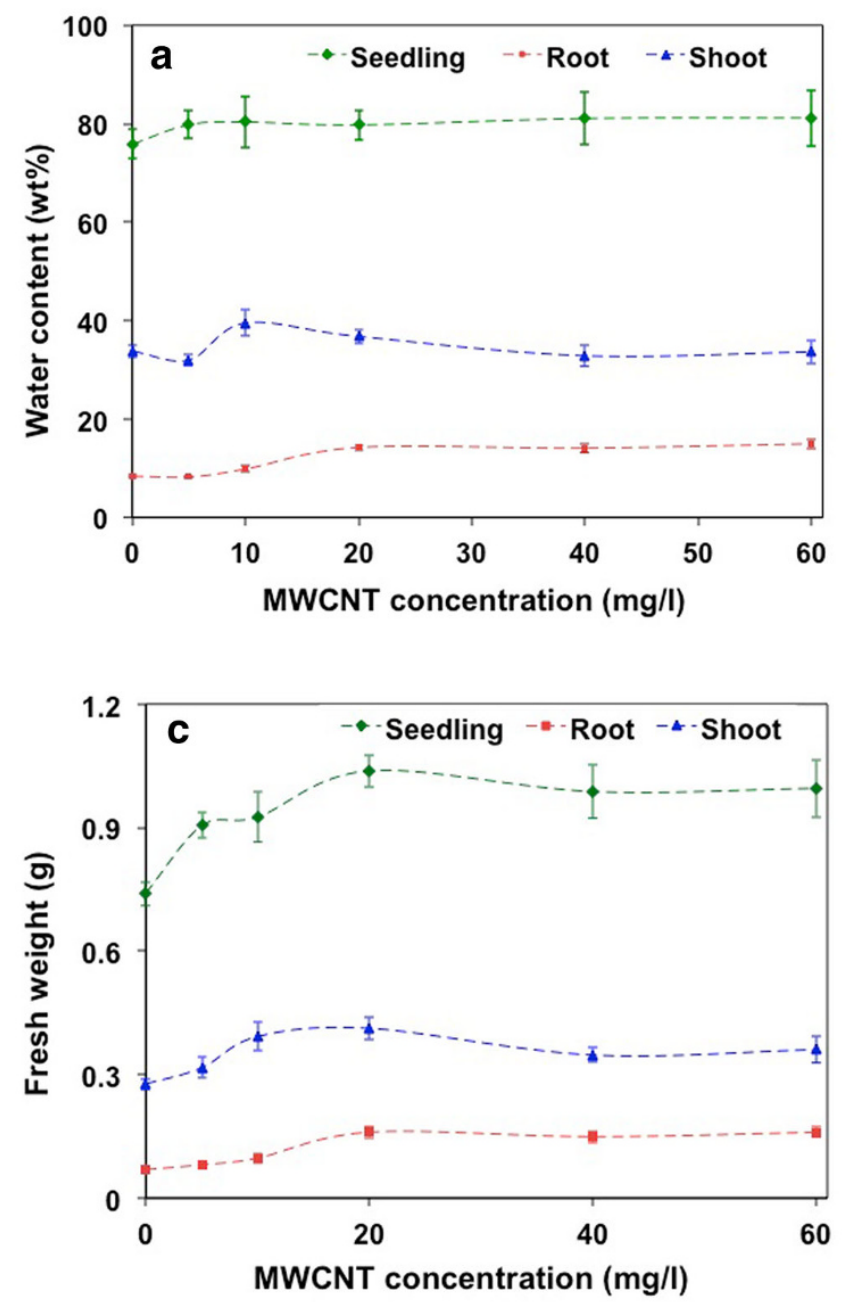

Fig. 1 Effect of multi-walled carbon nanotubes (MWCNT) on the 7-day germination and growth of maize in the BA medium (see Table 1). $(n=18)$. (a) \% Water content of the whole seedling, the root and the shoot versus the [MWCNT]. (b) Root (RL) and shoot lengths (SL) with respect to their \% water contents (c) fresh weights for the SLs, the increase with shoot \%water was weaker and more "noisy".

Figure $2 \mathrm{a}-\mathrm{d}$ refer to the time $(t)$-series analysis at the fixed [MWCNT] of $20 \mathrm{mg} / \mathrm{l}$ (EXP-2). With the exception of one pair of data points, the seedling DWs for the MWCNT treated medium and the control (Fig. 2a), are not different statistically. The seedling FWs on the other hand increase as they should as in any growing organism. Figure $2 b$ re-affirms the higher water intake by the seedling in the presence of the MWCNT and indicates a decreasing rate of intake with time (logarithmic fits). Figure $2 \mathrm{c}$ in a sense is a "corollary" of Fig. 1b: the [MWCNT] is fixed but the observations correspond to the different growth periods [the seedling water content is a parametrically time-dependent quantity (Fig. 2b)], showing the variation of the root and shoot lengths with water content where the
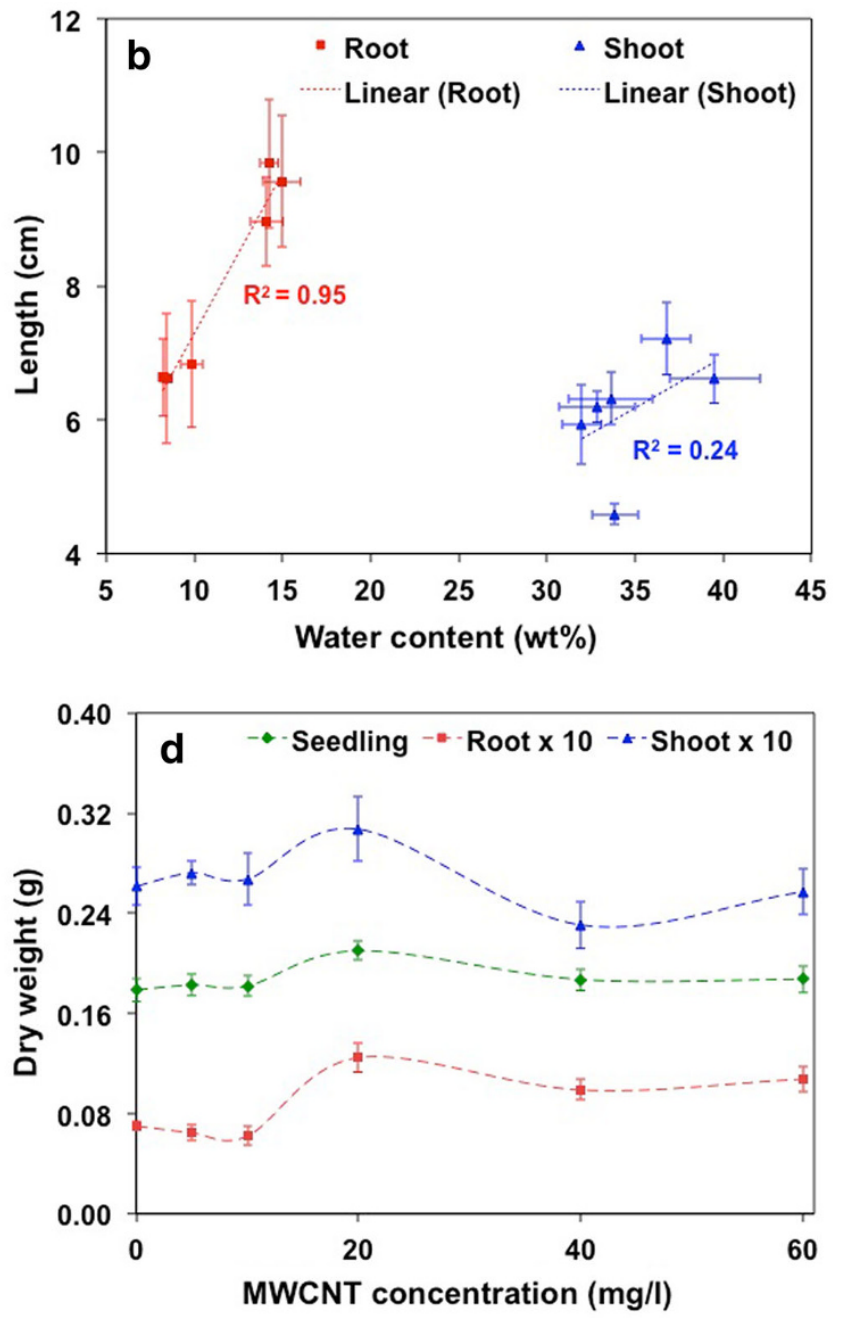

(FW) and (d) dry weights (DW) of the different morphological parts versus the [MWCNT]. The root and shoot DWs have been multiplied by 10 to accommodate them in the same graph. The dashed lines in $(\mathbf{a}, \mathbf{c}, \mathbf{d})$ are splined connectors only. The dotted lines in (b) are the linear regression fits to approximately quantify the trends

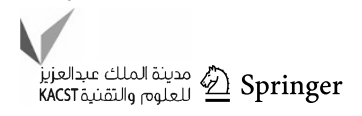


latter is the key entity affected by the MWCNT (Fig. 2b). The plots in Fig. 2d were constructed to test the validity of the universal allometric power-law scaling of biology (West and Brown 2005; Enquist 2002) for the water content versus the root and shoot FWs in the context of MWCNT mediated growth, given that water is the principal actuator of germination and metabolism (MacDonald 2007; Taiz and Zeiger 2006).

The seedling water content in the agarose medium (Fig. 3a) of EXP-3 did not show a high degree of variation with respect to the control except when ferrous chloride alone (A2) or ferric chloride with MWCNT (A5) were added. The trend of the water content was mirrored in the root and shoot DWs (Fig. 3b) albeit more sensitively particularly for the shoot.

The elemental analysis of the seedlings of EXP-1 by pEDXRF (Fig. 4) yielded the tissue concentrations of the macro and micro mineral nutrients of the 3rd and 4th
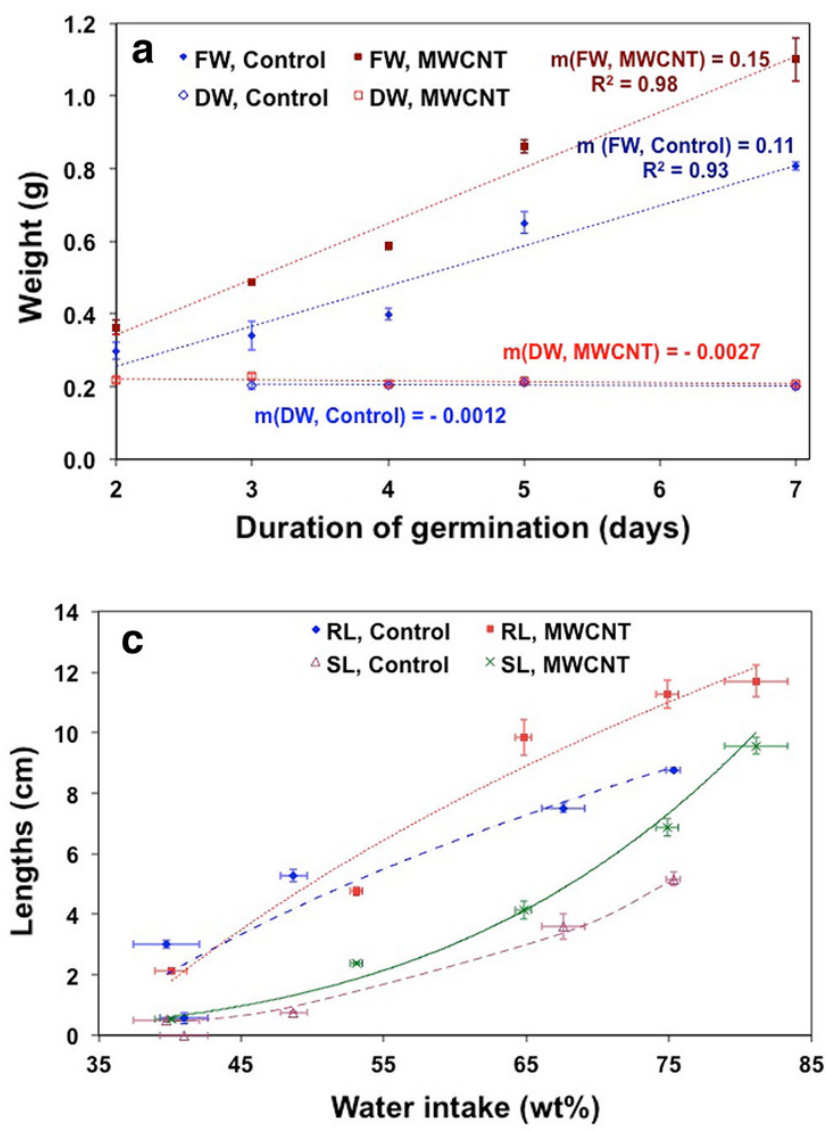

Fig. 2 Variation of growth indices with duration of germination of maize seedlings grown in BA with/without (三control) $20 \mathrm{mg} / \mathrm{l}$ MWCNT. (a) FW and DW. The dotted lines are the linear regression fits with the slopes $(\mathrm{m})$ and correlation coefficient $\left(R^{2}\right)$ values as shown. (b) \% Water intake. The dotted lines are the logarithmic fits. (c) RL and SL with respect to \%water intake by the seedling. The dotted and the dot-dashed lines are the logarithmic fits to the RL, the solid and dashed lines are the power-law fits of the SL, for the periods $\mathrm{f}$ the Periodic Table. With the exception of $\mathrm{Cl}, \mathrm{K}$, $\mathrm{Ca}$, and $\mathrm{Fe}$, there is no statistically significant difference between the Control and the lowest [MWCNT] of $5 \mathrm{mg} / \mathrm{l}$. Only in the cases of $\mathrm{Cl}$ and $\mathrm{K}$ does the Control have the highest concentration of the element in the plant tissue. As the MWCNT is introduced, the tissue $\mathrm{Cl}$ and $\mathrm{K}$ concentrations fall till 10 and $20 \mathrm{mg} / \mathrm{l}$ of [MWCNT], respectively then gradually level off. For all other elements the tissue elemental concentrations display a minimum at $10 \mathrm{mg} / \mathrm{l}$ [MWCNT] except for $\mathrm{Ca}$ and $\mathrm{Fe}$ for which the minima occur at 40 and $20 \mathrm{mg} / \mathrm{l}$. After the minimum, the tissue elemental concentration rises and maintains a level that is not statistically significantly different between the different higher [MWCNT], except for Ca.

Figure 5 shows the pEDXRF elemental analysis of the seedlings of EXP-3 for the same set of macro and micro mineral nutrients. Other than $\mathrm{Ca}, \mathrm{Fe}$, and $\mathrm{Cl}$, tissue concentrations of all elements of the set are unchanged
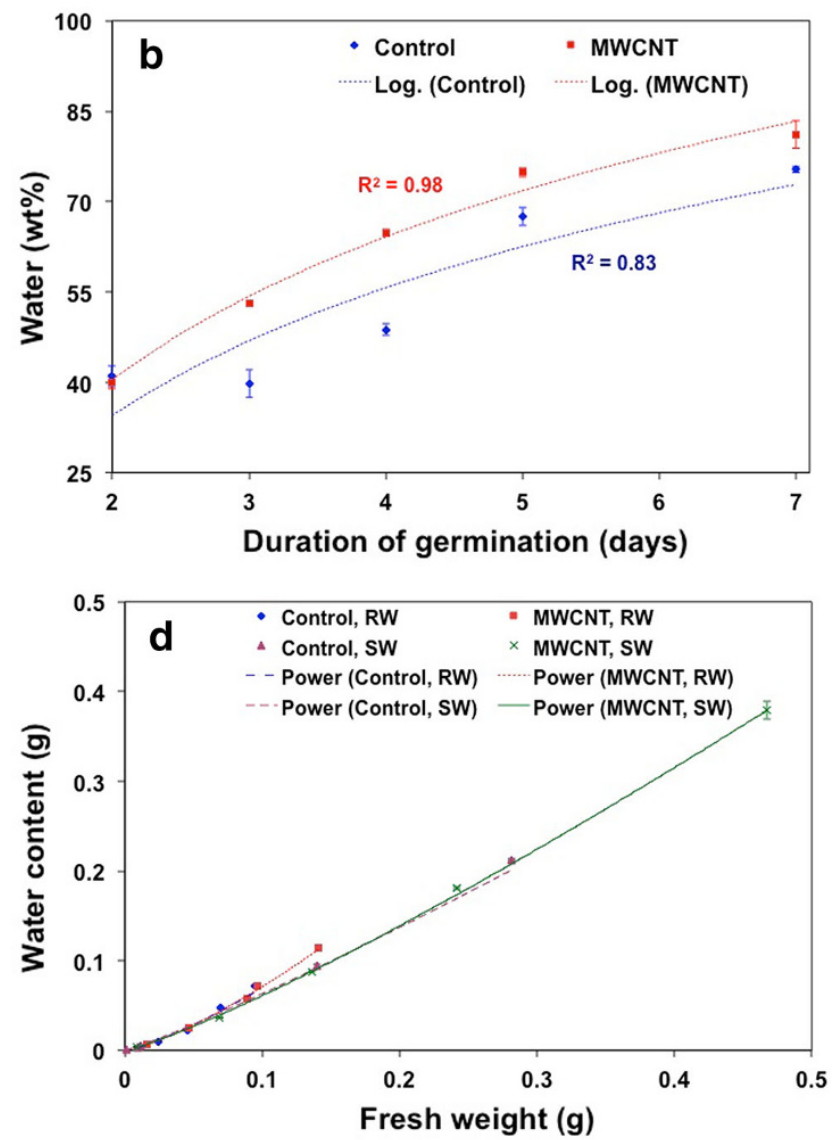

MWCNT and Control. The $R^{2}$ values respectively are $0.96,0.88$, $0.98,0.98$. (d) Test of universal allometric power law scaling of biology. The y-axis is the net water content of the shoots (SW) and roots (RW), respectively. The dotted, dot-dashed, solid and dashed lines are the power-law fits to the RW and SW with and without the MWCNT, respectively. The $R^{2}$ values in sequence are, $0.998,0.986$, $0.999,0.998$ 

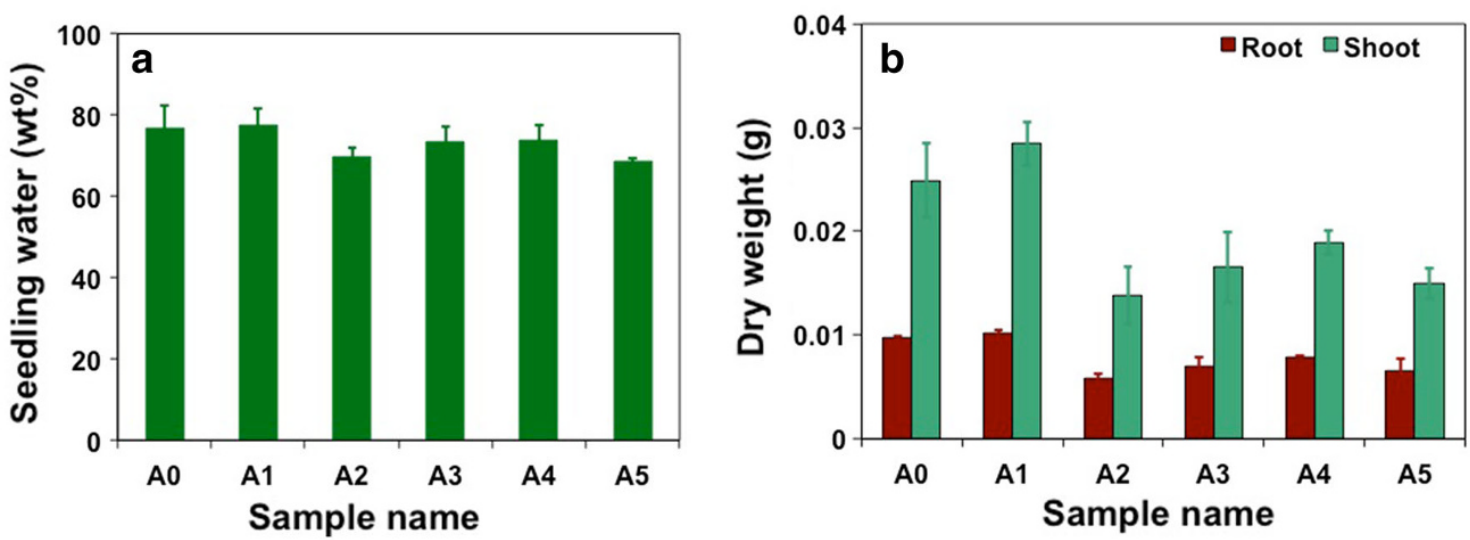

Fig. 3 Seedlings grown in agarose media (see Table 2). $(n=40$ or 41$)$. (a) Variation of \%water of the seedling. (b) Root and shoot DWs
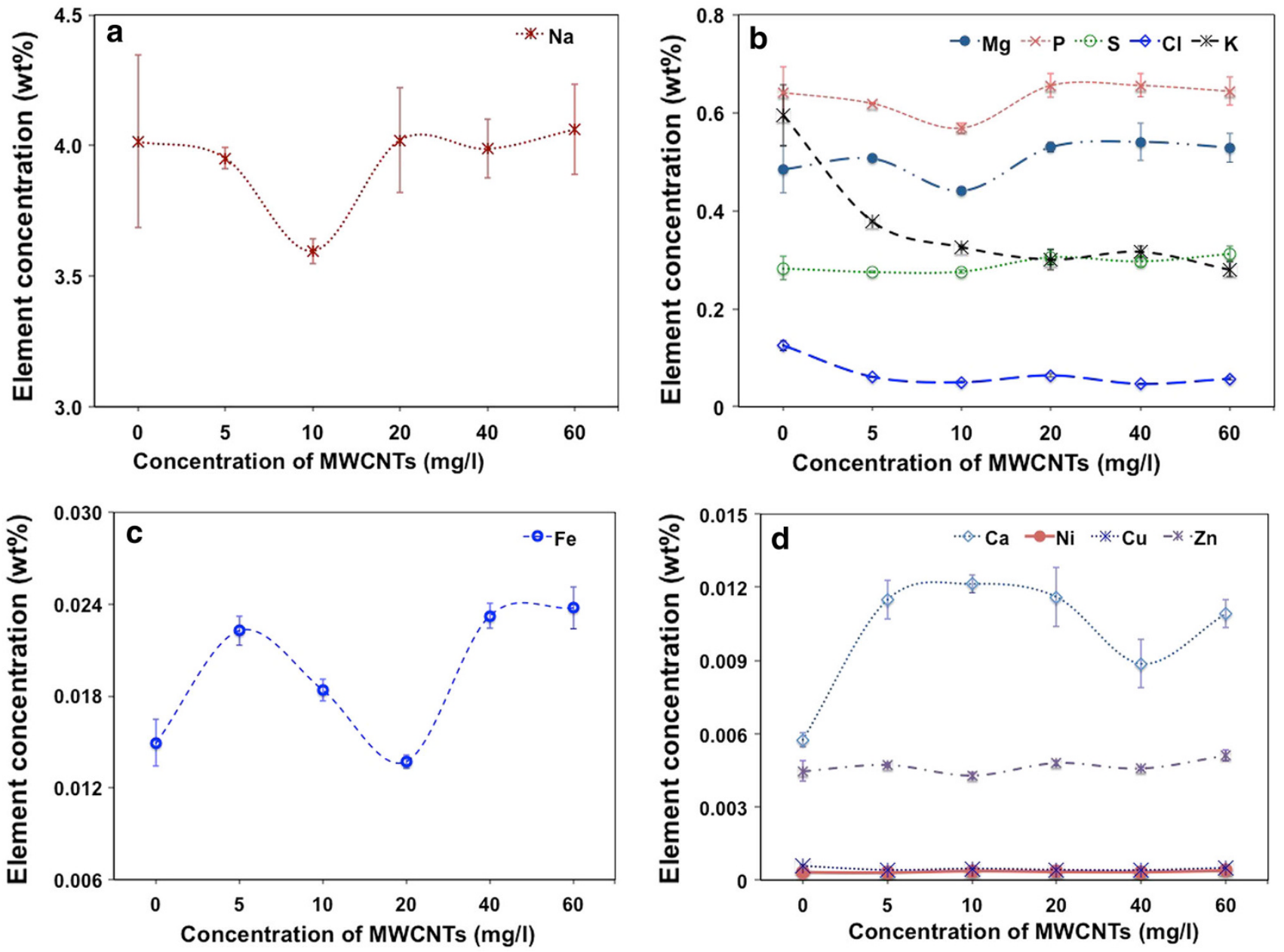

Fig. 4 Elemental concentrations of the seedling samples corresponding to Table 1 obtained using polarized energy dispersive X-ray fluorescence spectrometry (pEDXRF)

regardless of the medium (Fig. 5b). This is expected because the agarose is nearly completely free of extraneous elements. Thus none of these elements can be brought into a significant measure from the external medium and therefore retain their intrinsic concentrations in the seedling for which the type of external medium is inconsequential. The difference in the $\mathrm{Cl}$ concentrations compared to the control show up in the media A3 

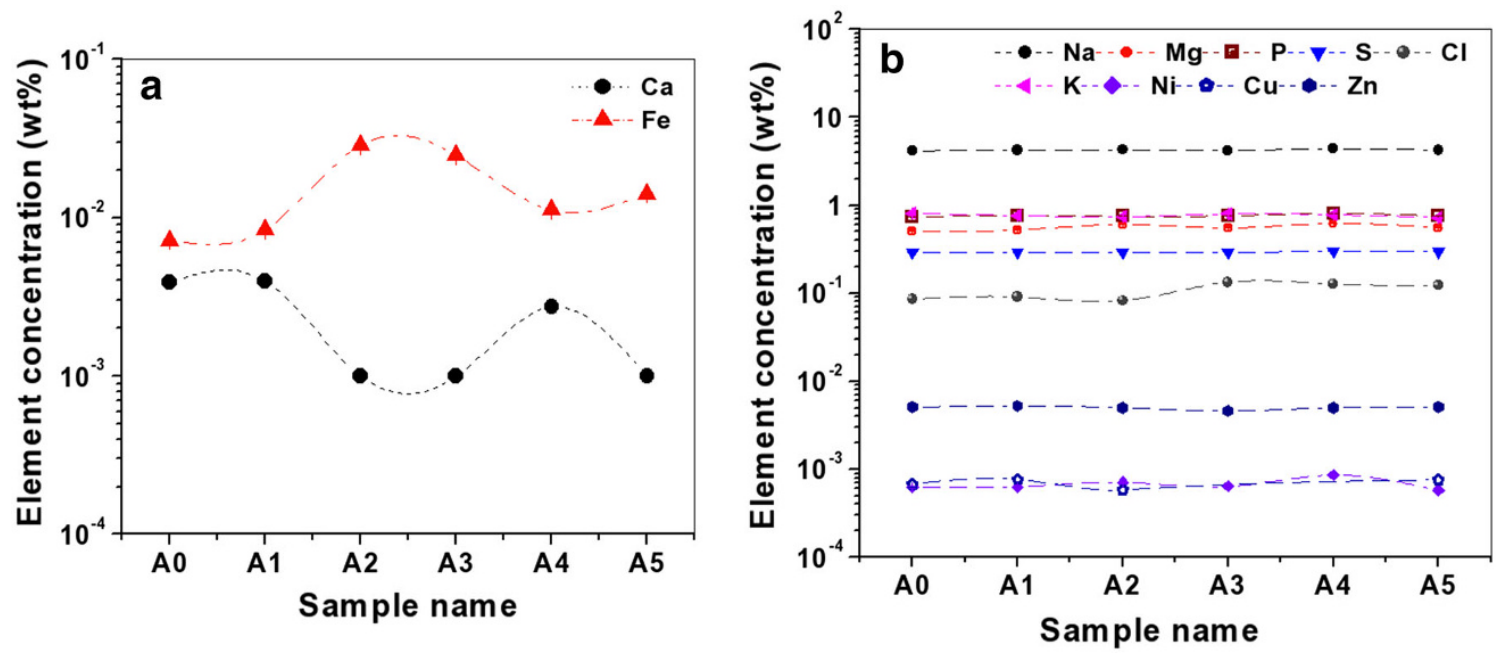

Fig. 5 Elemental concentrations of the seedling samples corresponding to Table 2 obtained using pEDXRF. The small errors bars are not visible, however the coefficients of variation averaged over all [MWCNT] are $0.1-3.2 \%$ for the different elements

$(\mathrm{Fe}(\mathrm{II})+\mathrm{MWCNT})$, A4 (Fe(III)) and A5 (Fe(III) + MWCNT). The most interesting cases are of the $\mathrm{Ca}$ and Fe concentrations whose trends are near mirror images of each other. Since $\mathrm{Fe}$ and not $\mathrm{Ca}$ is present in the agarose and $\mathrm{Ca}$ only in the cell walls and intracellular compartments of the seedling, the non-constant concentration of $\mathrm{Ca}$ is likely a result of the $\mathrm{Fe}, \mathrm{Ca}$ interaction in the media and plant cells with mediation by the MWCNT. Although $\mathrm{Fe}$ is frequently used as a catalyst in the manufacture of CNTs, the closeness of the Fe concentration of the agarose medium containing the MWCNT (A1) with the $\mathrm{Fe}$ for the plain agarose medium (A0, control) as well as the purity of $>95 \%$ of the MWCNT used in this work makes contamination by the Fe from such a source unimportant.

Figure 6 shows the SEM images of the black-layer region corresponding to the treatments in the agarose medium (EXP-3). The most interesting feature is how the pure MWCNT modifies the surface topography (Fig. 6c) of this region and how the relatively high ionic concentrations of iron and chloride ions in the medium change the capacity of the MWCNT to modify this surface topography (Fig. 6d, e).

\section{Discussion}

Water content and growth indices (EXP-1)

The region of the black-layer of the pericarp/seed-coat where the radicle emerges is the first point of entry of water and oxygen molecules in the germinating maize seed (McDonald et al. 1994). The MWCNTs suspended in the gel medium as well as the dissolved ions and other species
$\left(\mathrm{Fe}^{2+}, \mathrm{Fe}^{3+}\right.$, and $\mathrm{Cl}^{-}$only, in the agarose gel media) would be carried along with the water and are likely to show their effects prominently here. In particular if the MWCNT can penetrate seed coats (Khodakovskaya et al. 2009) they would find it the easiest to do in this part because of the propensity of this region to allow the entry of external molecules. Transmission electron microscopy (TEM) and Raman spectrometry (Villagarcia et al. 2012; Mondal et al. 2011; Tripathi et al. 2011) have shown the penetration into seedling tissue (except the shoots) by the MWCNT. The difference that the MWCNT make to the surface of the seed's black-layer is evident in the comparison between the Fig. $6 c$ and b. A high degree of porosity and a partial dismemberment of the surface structure are seen upon introduction of the MWCNT (Fig. 6c). Such pores would greatly facilitate the entry of water, nutrients and oxygen as well as the dispersed aqueous phase MWCNT themselves into the germinating seed. It is well known that scarification aids the germination of seeds. Visually, the appearance of the effect of the MWCNT at the black-layer region (Fig. 6c) seems akin to scarification at the microscopic scale.

The seed body and root are directly in contact with the gel medium. The higher water permeation upon introduction of low [MWCNT] due to the aforesaid pores is likely the cause for the higher uptake of water implicit in the Figs. 1a, c, and 2a, b. Higher [MWCNT] would be logically expected to increase the penetrability and hence the water content. However, the water contents are seen to follow the trends described in "Results, Fig. 1". Figure 6c indicates numerous large pores at the [MWCNT] of $20 \mathrm{mg} /$ 1 (size of the order of $\mu \mathrm{m}$ ), thus the putative increase of porosity with $[\mathrm{MWCNT}] \geq 20 \mathrm{mg} / \mathrm{l}$ is not expected to substantially enhance the water content. Furthermore, at the higher [MWCNT] in the medium, the nanotubes would 

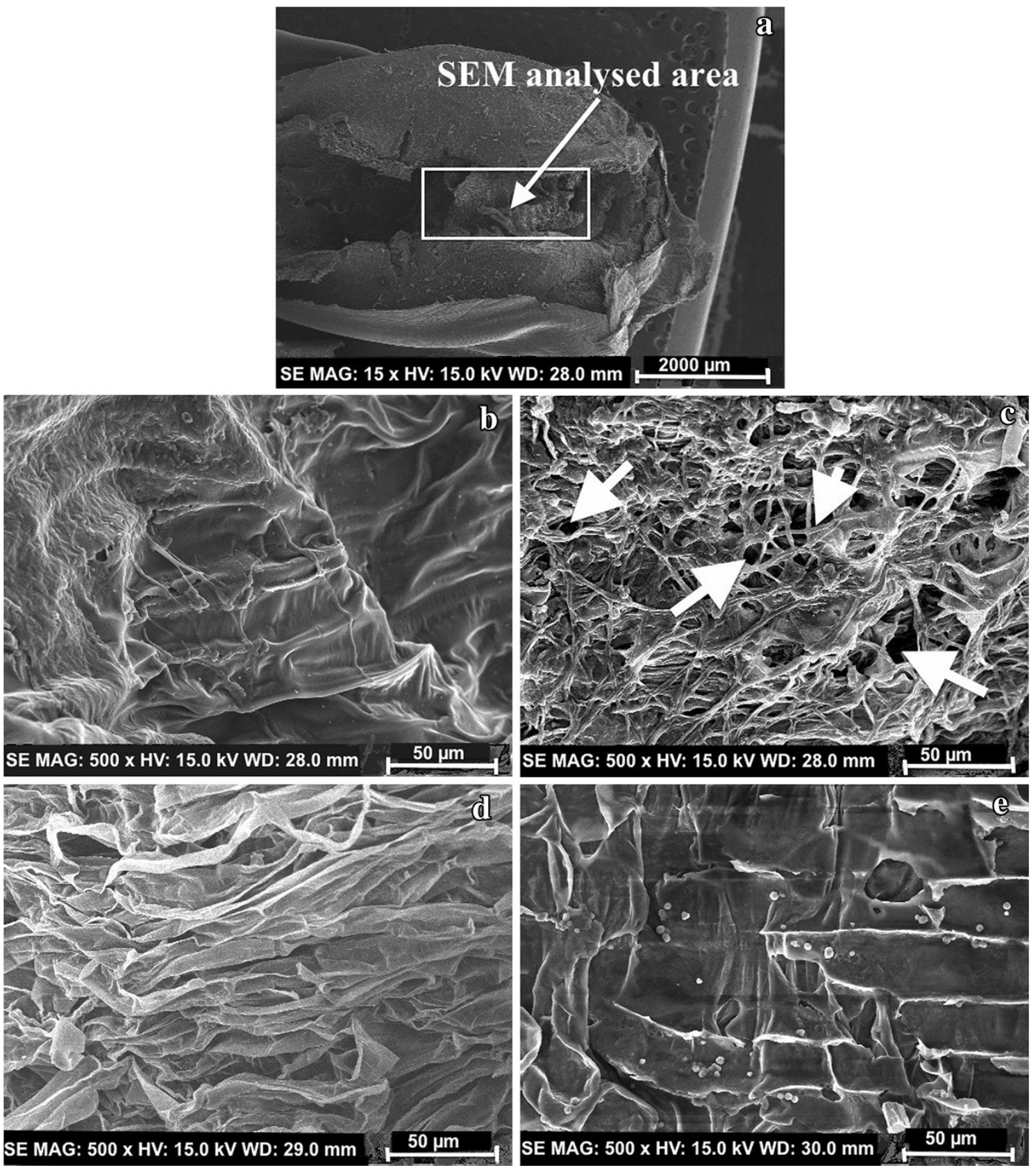

Fig. 6 SEM images of the topography of the black-layer region of the seed-coat of the 7 day germinated maize seedling grown in agarose gel with some of the additives of Table 2. (a) The analyzed area near the black-layer. (b) Control medium. (c) MWCNT

(20 mg/l) medium; the white arrows indicate the pores. (d) $\mathrm{Fe}(\mathrm{III})$ $\left(3 \times 10^{-4} \mathrm{M}\right)$ medium (A4) (e) Fe(II) $\left(3 \times 10^{-4} \mathrm{M}\right)+$ MWCNT $(20 \mathrm{mg} / \mathrm{l})$ medium (A3)

aggregate more (particularly in the absence of induced exfoliation) resulting in the diminution of functionality such as their capacity to increase water delivery to the parts in contact with the medium. These factors may account for the scant variation of the \%water at the $[\mathrm{MWCNT}] \geq 40 \mathrm{mg} / \mathrm{l}$. The shoots are not immersed in the medium so water enters the shoot as for all plants by capillary action driven by the root water pressure. Aggregation of the MWCNT in the extracellular or intracellular

spaces within the root, that is likely to scale with the [MWCNT] (Vaisman et al. 2006), would impede this normal upward flow and affect the physiology of shoot water transport at the higher [MWCNT]. The initial formation of intracellular aggregates within the root of gram plants even by the wsMWCNTs, has been observed (Tripathi et al. 2011). Oxidative damage at the higher [MWCNT] concentrations (Lin et al. 2010) consequent to the penetration of the plasma membrane of the seedling

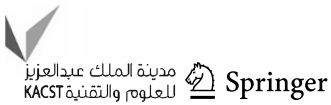


cells by the CNT (Liu et al. 2009) may also play a role in the reduction of the water content and FW of the shoot (Fig. 1c, "Results") at the higher [MWCNT]. The combination of the facilitation of water entry at low [MWCNT] and but its 'obstruction' at higher [MWCNT] makes the trace of the shoot water content pass through a broad maximum at the [MWCNT] of $\sim 20 \mathrm{mg} / \mathrm{l}$.

The root length ("Results, Fig. 1") shows a clear $\left(R^{2}=0.95, p<0.05\right)$ and steep dependence on the quantity of water imbibed which in turn, as seen in the foregoing, is correlated with the [MWCNT]. Water increases the cell turgor pressure that triggers cell elongation and the production of hormones [e.g. gibberellic acid (GA) and auxin] that promote root growth (MacDonald 2007; Taiz and Zeiger 2006). The weaker and noisier increase $\left(R^{2}=0.24, p<0.05\right)$ of shoot length with water content ("Results, Fig. 1") may be a consequence of two factors. While water uptake induces shoot growth also through the aforesaid physiological processes (MacDonald 2007; Taiz and Zeiger 2006), the uptake by the shoot is indirect and dependent on the upward water transport by the root. The latter would be affected by the [MWCNT] in the manner described in the previous paragraph. Additionally, other independent factors such as light intensity are also involved in shoot growth (Taiz and Zeiger 2006). The combination of the different factors produces a noisier and weaker response of the shoot length to just the water content.

Figure 4 shows that for most nutrients (except $\mathrm{Cl}$ and $\mathrm{K}$ ) the concentrations rise at [MWCNT] $>10 \mathrm{mg} / \mathrm{l}$ (at $>0 \mathrm{mg} / \mathrm{l}$ [MWCNT] in the case of $\mathrm{Ca}$ and $\mathrm{Fe}$ ). The [MWCNT] of $\sim 20 \mathrm{mg} / \mathrm{l}$ is also the concentration where the water content for the different parts, particularly the root, reaches high values/maximum (Fig. 1a). The rise in imbibed water brings in more mineral nutrients from the medium and also signals enhanced enzyme and hormone activity (MacDonald 2007 and Private Communication Feb. 2013), factors that are expected to benefit tissue development. This consequence of the water absorption is seen in the rise of the relative concentrations of several mineral nutrients, particularly Ca (Fig. 4), and in the DWs (Fig. 1d) where a maximum is reached at the [MWCNT] of $20 \mathrm{mg} / \mathrm{l}$. Root and seedling water content hold nearly steady after $20 \mathrm{mg} / \mathrm{l}$ [MWCNT] (Fig. 1a) so the water borne increase of mineral nutrients is not expected to increase after this concentration, which indeed is broadly the case (Fig. 4). However, increased [MWCNT] is a possible source of oxidative cell damage as discussed in the preceding as well as causing perforations in the seed epidermal matrix (Fig. 6c). These factors may impact the metabolic activity adversely resulting in a decline of the root and shoot DW with increased [MWCNT].

Thus the benefits of the MWCNT in this work are observed for the lower end of the concentration range
( $\leq 20 \mathrm{mg} / \mathrm{l}$ ). Toxicity effects (Lin and Xing 2007; Lin et al. 2010) diminish the usefulness of the MWCNT to the plant at higher concentrations. The effect of the MWCNT on plant health therefore appears to be one of chemical hormesis, a phenomenon that has been observed for normally toxic substances in areas such as toxicology and homeopathy (Calabrese and Baldwin 2000).

Time series analysis (EXP-2) of water absorption and growth indices

The overall tendency of the data for the FWs in Fig. 2a may be qualitatively inferred from the slopes of the trend lines. The magnitudes of the slopes indicate that the FW in the MWCNT medium increases $\sim 1.4$ times faster than the control. These results are reasonable given the observation of the MWCNT aiding water imbibition ("Water content and growth indices (EXP-1)") and due to FWs being mostly water. Likewise the approximate overall tendency of the seedling DWs in Fig. 2a may be inferred from the trend lines whose slopes differ statistically $(\leq 70 \%$ confidence level). The DWs in both the MWCNT and control media show a decreasing trend with time (negative slope) in keeping with the physiology of seed germination before photosynthesis sets in (MacDonald 2007). The magnitudes of the slopes show that the DW of the MWCNT treated seedlings decrease at almost double the rate of the control seedlings. This would indicate that the higher water imbibition caused by the MWCNT triggers a higher rate of metabolic activity and respiration through the activation of enzymes and hormones (MacDonald 2007) leading to a faster DW decline.

Water enters the cells of the gel submerged parts of the seedling by passive diffusion driven by the difference of the water potential across the gel-seedling barrier (Taiz and Zeiger 2006). As more water fills the cellular spaces with time, the magnitude of the potential difference decreases resulting in a lowered rate of water entry or a saturation effect, in keeping with Fick's second law of diffusion (Taiz and Zeiger 2006). The saturation effect shows up clearly for both the control and the MWCNT treated seedling in Fig. 2b. The MWCNT treated seedlings consistently absorbed more water than the control. To qualitatively indicate the non-linear or saturative behavior, the data were fitted to $\log$ functions that were seen to produce the most acceptable fits. The MWCNT data produced a better quality of fit $\left(R^{2}\right.$ value). In fact the better statistics of all data sets concerning the MWCNT is a feature that stands out. Statistically, the degree of orderliness in the data values (precisions) is indicated by how low the average coefficient of variation ( $\mathrm{CV}$ for the set) is. The $\mathrm{CV}$ values for the water contents and growth indices studied in this experiment for the MWCNT treated seedlings were 
consistently lower, of the order of $0.5-0.7$ times the values for the corresponding control data sets. Molecular dynamics simulations of water in CNTs show that CNTs act as molecular channels for water where the hydrophobicity confines the water molecules to ordered files of polarized chains and moves them in a pulsatile fashion (Alexiadis and Kassinos 2008). In an attempt to investigate this phenomenon experimentally, Tripathi et al. (2011) have shown that their wsMWCNT aligned themselves in the vascular bundles of the roots of gram plants and enhanced plant water uptake possibly by the ensuing channeling action. It is possible then that the enhanced water uptake shown in this work and the orderliness of the behavior consequent to MWCNT application, might also result from a similar alignment and channeling in contrast to the normal random thermal motion of the water molecules in the control.

In Fig. $2 \mathrm{c}$ the rate of root length increase diminished with the increase of seedling water content (logarithmic fit) which at first seems aberrant. However, the in vitro growth constrains the downward elongation of the root-a situation that is not encountered in field plants. Such a confinement is less rigorous for the upward elongation of the shoot. For both media, the SL rapidly increases (power-law fit) as a consequence of water imbibition and phototropism. However, one observes that for the same water content (i.e. same values of \%water on the fitted curves), the RL and SL for the MWCNT case are higher than for the control. The same water content implies that all water related growth factors would be invariant. Thus, MWCNTs at low concentrations seem to interact with the seedling cells at more levels than just the facilitation of water delivery, to enhance growth.

In a landmark study (West and Brown 2005; Enquist 2002) West, Brown and Enquist (WBE) have argued that mass is the prime determinant of variation in physiological behavior when different organisms are compared over many orders of magnitude. Scaling with mass then, they show follows a simple power-law,

$Y=Y_{0} \cdot M_{b}^{b}$

where $Y$ is some observable metabolism-related quantity, $Y_{0}$ is a normalisation constant and $M_{\mathrm{b}}$ is the mass of the organism. At the core of this allometric scaling law is the axiom that the exponent $b$ takes on a limited set of values, typically simple multiples $(n)$ of $1 / 4$. For the fluid volumes in trees (Enquist 2002), $b$ is seen to be $25 / 24=4.17(1 / 4)$ or rounded to the nearest integer, it is $4 \cdot(1 / 4)$. We have seen in the current experiment that the water content is a crucial metabolic driver in germination and early growth and is affected by the presence of the MWCNT. In Fig. 2d we explore the question of whether the same one-fourth behavior carries over to seedlings in the artificial environment of the MWCNT and if different, how much different from the control. The fluid being principally water, its weight $(\mathrm{g})$ would approximate closely the volume $(\mathrm{ml})$ at room temperature and pressure. Figure $2 \mathrm{~d}$ shows that the power-law fitted the experimental data of the variation of the water contents (in $g$ ) in the morphological parts versus their FWs, rather well ( $R^{2}$ values as indicated). The plots for all parts whether or not treated by the MWCNT are nearly identical with only small variations in the exponent values. The values of $\left\{Y_{0}, b\right\}$ for the root (control), root (MWCNT), shoot (control), shoot (MWCNT) were respectively, $\{1.354,1.288\},\{1.527$, $1.331\},\{0.829,1.118\},\{0.932,1.184\}$. Rounding the $b$ values to the nearest integral multiple of $1 / 24$ we get $b$ as, $31,32,27$, and 28 times the fraction $1 / 24$, respectively for the sequence. An average of these multiples of $1 / 24$ rounded to the nearest integer yields $(30 \pm 2)$. In other words the fluid (water) volume obeys Eq. (1) with the exponent as $\sim 30 / 24$ or $5(1 / 4)$. (N.B. If the shoot alone is considered, the exponent is closer to $25 / 24$, being $\sim 27$ or 28 times 1/24). Thus the seedling follows the expected universal scaling law just as the giant trees, many orders of magnitude its size, do. The perturbation brought in by the xenobiotic MWCNT to the seedling's physiology does not make it deviate from the law. The value of $b$ however is one integer unit in excess of the $b$ value typical for trees showing that water constitutes the masses of these young plants more than for the older trees. WBE point out that it is not so much the closeness of the value of $b$ to $n \cdot 1 / 4$ is but rather its approximation over a great range of sizes and conditions (e.g. our seedlings versus giant trees) that is important and that the residual variation may throw light on the secondary factors that cause the deviation from the exact $n \cdot 1 / 4$ value. Seen in this light, we note that the $b$ for the MWCNT cases are consistently somewhat higher than for the control. We posit that a detailed logging of the values of $\mathrm{b}$ in such types of experiments for various $Y$ may empirically allow the quantification (and thereby prediction) of the effects of the given MWCNT.

\section{Elemental concentrations in seedling tissue (EXP-1 and EXP-3)}

The salts of the mineral macro and micronutrients of the BA in the aqueous gel medium present the elements principally as ions or polar molecules to the plant tissue. The flow of water into the plant (principally the root) facilitated by the MWCNT as seen in the foregoing, brings in the dissolved ions where the inflow varies as shown in Fig. 1a. However the MWCNT surfaces in the medium may attach the ions through the ion-transient dipole (ITD) interactions between charged/polar species and the $\pi$-electron clouds in the CNT molecular structure as has been documented in the 
literature (see for example, Beu 2011). The strength of these interactions will vary with the type of ion and CNT, CNT concentration, state of CNT aggregation etc. in a complex way. Hence the profile of the elemental concentrations (Fig. 4) is likely the result of a trade-off between these two contrary forces-increase by water inflow versus retention (adsorption) by the medium's MWCNT. We proceed to describe the specific cases vis à vis this probable mechanism.

For the maximally electro-positive and negative ions in the medium $\left(\mathrm{K}^{+}\right.$and $\left.\mathrm{Cl}^{-}\right)$the introduction of the different [MWCNT] only serves to decrease their concentrations up till a broad minimum after which the concentrations flatten out (Fig. 4b). The ITD interaction is likely to dominate over water inflow for these highly electro-active ions restricting their ingress into the seedling. A saturation is seen at $[\mathrm{MWCNT}] \geq 20 \mathrm{mg} / \mathrm{l}$ possibly because of increased MWCNT aggregation that reduces the surface area despite the increase of [MWCNT]. For other ions except $\mathrm{Fe}$ and $\mathrm{Ca}$, the attractive force appears to dominate up till $\sim 10 \mathrm{mg} / \mathrm{l}$ of MWCNT. At [MWCNT] $\geq 20 \mathrm{mg} / \mathrm{l}$ the increased water inflow (Fig. 1a) dominates, increases the elemental concentrations, which then level off as a consequence of the plateau in the water content (Fig. 1a) and the MWCNT aggregation. In the case of $\mathrm{Fe}$ the [MWCNT] of $5 \mathrm{mg} / \mathrm{l}$ causes a sharp rise but for $[\mathrm{MWCNT}]>5 \mathrm{mg} / \mathrm{l}$ the interplay between the adsorptive forces, the increase of water inflow and MWCNT aggregation as for the other ions, makes the profile pass through a minimum $(20 \mathrm{mg} / \mathrm{l})$ with the subsequent rise and leveling off. The case for $\mathrm{Ca}$ is similar except that its high concentration spans a broad plateau up till $20 \mathrm{mg} / \mathrm{l}$ and the minimum occurs for the higher [MWCNT] of $40 \mathrm{mg} / \mathrm{l}$. Increased water transport finally prevails (at [MWCNT] $60 \mathrm{mg} / \mathrm{l}$ ) increasing the $\mathrm{Ca}$ concentration but lingering adsorptive effects likely make the value less than at the plateau region. These examples show the specific nature of the ion (polar molecule)-CNT interaction and the role of water flux in the overall ionic transport into the seedling.

We have seen that $\mathrm{Fe}$ and $\mathrm{Ca}$ are the two nutrients that show some of the most sensitive responses to the MWCNT (Fig. 4c, d). EXP-3 in the agarose medium was constructed to inspect the behavior of $\mathrm{Fe}$ without the interference of the other ions at the [MWCNT] of $20 \mathrm{mg} / \mathrm{l}$ where the CNT displays a strong adsorptive effect (Fig. 4c) and where water inflow is relatively high (Fig. 1a). We hypothesize that the strong CNT-Fe interaction if guided by the ITD would be affected by the charge state of the Fe; hence a difference would presumably show up between $\mathrm{Fe}^{2+}$ and $\mathrm{Fe}^{3+}$ introduced at the same concentrations. The plant's own preferential absorption of $\mathrm{Fe}^{2+}$ would also factor into the overall response. In plants typically the enzyme iron reductase secreted by the root plasma membrane, reduces
$\mathrm{Fe}^{3+}$ to $\mathrm{Fe}^{2+}$ to facilitate $\mathrm{Fe}$ uptake (Taiz and Zeiger 2006). Figure 5a shows that the plant absorbs distinctly more $\mathrm{Fe}^{2+}$ (A2) than $\mathrm{Fe}^{3+}$ (A4) in the absence of the MWCNT. When the MWCNT is introduced, the seedling $\mathrm{Fe}$ concentration from $\mathrm{Fe}^{2+}$ is slightly reduced (A3 lower than A2, Fig. 5a) despite the better water delivery in A3 compared to A2 (Fig. 3a) possibly because of the adsorption of $\mathrm{Fe}^{2+}$ onto the MWCNT in the medium. In the case of $\mathrm{Fe}^{3+}$ with the MWCNT however, the Fe concentration within the plant slightly increases (A5 more than A4, Fig. 5a) despite the reduced water delivery in A5 compared to A4 (Fig. 3a). If the $\mathrm{Fe}$ were to remain as $\mathrm{Fe}^{3+}$ then the combined effect of the plant's low-affinity for $\mathrm{Fe}^{3+}$ and the higher electrostatic attraction by the electron cloud in the MWCNT for the higher positive charge, would have reduced the plant $\mathrm{Fe}$ concentration further. The contrary observation, viz. the increase, may then possibly be due to the reduction of $3+$ to $2+$ by the CNT's electronic density thereby facilitating the uptake of Fe by the plant. The differences in Fe concentrations and seedling \%water contents cited here are statistically different at $95 \%$ confidence level for the concentrations and at 80-90\% confidence level for the \%water.

$\mathrm{Ca}$ is present in the cell wall matrix of the seedling largely in the binding heteropolysaccharide, pectin, as calcium pectate (Taiz and Zeiger 2006). The addition of MWCNT alone (A1) makes no change to the Ca content in the seedling, as it should be. However, when $\mathrm{Fe}^{2+}$ is added either alone (A2) or with the MWCNT (A3) a relatively high reduction in the $\mathrm{Ca}$ content in the seedling takes place in a diametrically opposite manner to the $\mathrm{Fe}$ contents (Fig. 5a). This suggests that $\mathrm{Ca}$ is cationically exchanged by the $\mathrm{Fe}^{2+}$ in the cell wall matrix leading to a reduction in the seedling $\mathrm{Ca}$ content. When $\mathrm{Fe}^{3+}$ alone is introduced, the $\mathrm{Ca}$ concentration rises markedly almost exactly mirroring the reduction of $\mathrm{Fe}$ content in the seedling tissue (Fig. 5a). The non-equivalent positive charges between $\mathrm{Fe}^{3+}$ and $\mathrm{Ca}^{2+}$ makes the replacement of $\mathrm{Ca}^{2+}$ less probable by cation exchange (e.g. in the bonds with the pectate groups (Taiz and Zeiger 2006). However, when the $\mathrm{Fe}^{3+}$ is introduced with the MWCNT, the Ca content falls, again mirroring the rise in $\mathrm{Fe}$ content (Fig. 5a). If the $\mathrm{Fe}^{3+}-\mathrm{CNT}$ interaction causes some reduction of the $\mathrm{Fe}^{3+}$ to $\mathrm{Fe}^{2+}$ which in turn exchanges with the $\mathrm{Ca}^{2+}$ in the cell wall matrix, it would explain these aforesaid observations. The $\mathrm{Ca}$ concentration profile therefore provides further support to the possibility of the CNT surface promoting the $\mathrm{Fe}^{3+}$ reduction. Calcium bound to pectin has a structural and hydrating role in the cell wall and also functions in cellular metabolic regulation and signaling (Taiz and Zeiger 2006). Excess heavy metals (e.g. the $\mathrm{Fe}^{2+}$ in EXP-3) are known to modify the stability of $\mathrm{Ca}$ channels causing an increase of cellular Ca fluxes (Manara 2012). Given these functions, 
one may expect that the possible efflux of $\mathrm{Ca}$ out of the seedling would reduce its DW and the water content, particularly of the root. This expectation is supported by the observations of Fig. 3 that show that the root DW and total \%water content are the least as compared to the Control for the samples that possess the least $\mathrm{Ca}$ concentrations (A2 and A5 of Fig. 5a).

The interdependence of the $\mathrm{Fe}^{2+} / \mathrm{Fe}^{3+}$ and $\mathrm{Ca}^{2+}$ concentrations is contrasted with the near constancy of the $\mathrm{Cl}^{-}$ concentrations where $\mathrm{Cl}^{-}$is the counterion to the $\mathrm{Fe}^{2+}$ and $\mathrm{Fe}^{3+}$ in the system. The rise of $\mathrm{Cl}$ in the plant tissue for $\mathrm{A} 3$, $\mathrm{A} 4$, and A5 as compared to A2 is probably due to the effects of higher water inflow in A3 and A4 (Fig. 3a) and the higher mole ratio of $\mathrm{Cl}$ to $\mathrm{Fe}$ in $\mathrm{FeCl}_{3}$ compared to $\mathrm{FeCl}_{2}$.

Water contents, DWs and surface topography of seedlings in EXP-3

The ratios of the \%water contents of (a) A0 to the Control in EXP-1 (b) AI to the [MWCNT] of $20 \mathrm{mg} / 1$ in EXP-1 are (a) $1.01 \pm 0.08$ and (b) $0.97 \pm 0.06$ which are both close to 1 . The ratio in (a) proves that there is no intrinsic difference between the two types of gel media in their ability to supply water to the seedling. The second ratio shows the water transport to the total seedling by the MWCNT at $20 \mathrm{mg} / \mathrm{l}$ behaves in the same manner in the two media.

The SEM image of the seed coat in the black-layer area in the medium $\mathrm{A} 4\left(\mathrm{FeCl}_{3}\right)$ shows little physical change as compared to A0 (control, Fig. 6b), except a more striated or puckered surface (Fig. 6d). The introduction of the medium A3 $\left(\mathrm{FeCl}_{2}\right.$ with the $20 \mathrm{mg} / \mathrm{l}$ MWCNT) also leaves the seed coat intact with apparently even less layer folding (Fig. 6e). These images are in contrast to Fig. 6c (A1 medium) that show seed coat perforation which has earlier been linked to more facile water delivery ("Water content and growth indices (EXP-1)" and "Time series analysis (EXP-2) of water absorption and growth indices"). The similar surface topographies of A3 and A4 suggest the seedling water contents in A3 and A4 might be close which in fact is the case (Fig. 3a). The more intriguing feature is the difference that the $\mathrm{FeCl}_{2}$ makes to the surface topography when the background concentration of the MWCNT is the same (Fig. 6e versus c). The ITD force alluded to before ("Elemental concentrations in seedling tissue (EXP-1 and EXP-3)") may result in the adsorption or localization of some of the $\mathrm{Fe}^{2+}$ (and other ions) on the surface of the MWCNT, thereby altering its functionality so that it cannot so effectively cause the surface pores on the black-layer of the seed-coat (Fig. 6e). Such an alteration of the surface chemistry of the MWCNT may affect its ability to deliver the metal ion $\left(\mathrm{Fe}^{2+}\right)$ to the seedling and perhaps may also affect water delivery since the CNT surface affects water conduction ("Time series analysis (EXP-2) of water absorption and growth indices"). A3 is seen to have a slightly lower $\mathrm{Fe}$ concentration than A2 (Fig. 5a). While the effect on the DWs by the treatment in A3 versus the one in A1, is strong (Fig. 3b: DWs of A3 lower than A1), the corresponding decrease in the \% water content in A3 relative to A1 (Fig. 3a) is less prominent although the difference is statistically significant $(\leq 80 \%$ confidence level). It is possible that the aforesaid change in MWCNT functionality may also affect its enhancement of germination and growth. DWs are a result of metabolic processes. In Fig. 3b we see that the metabolic processes that contribute to root and shoot DWs were the highest for the pure MWCNT treated seedlings. The introduction of $\mathrm{FeCl}_{2}$ and $\mathrm{FeCl}_{3}$ only depreciated the DW enhancement by the MWCNT.

Overall the findings of EXP-3 suggest that the pristine MWCNT (medium A1) are more potent for seedling growth than MWCNT in media with high concentrations of ions or polar groups.

\section{Summary and conclusion}

We have shown that the germination of maize seedlings is affected by the MWCNT in a concentration dependent manner: lower concentrations are beneficial, higher ones prove relatively detrimental in what appears to be chemical hormesis. At the concentration of $20 \mathrm{mg} / \mathrm{l}$, the pristine MWCNTs increased the growth indices and the water contents of the morphological parts, most prominently for the root, which was directly in contact with the medium. Higher MWCNT concentrations were less effective possibly because of increased CNT clumping which impacted their potency and/or the toxicity of high MWCNT concentrations. The latter was more marked for the shoot.

The growth and water imbibition rates were found to be higher with the application of the MWCNT (at $20 \mathrm{mg} / \mathrm{l}$ ). However, for the same water contents the lengths were higher for the MWCNT plants indicating the benefit of the low dose of the MWCNT to the physiology in more ways than only water delivery. The consistently high statistical orderliness of the MWCNT measurements was conjectured to support the prevalent idea of the property of CNTs to act as molecular water channels. The $20 \mathrm{mg} / \mathrm{l}$ MWCNT was found not to cause departures from the universal allometric scaling law of biology applied to plant water volumes, indicating that as a xenobiotic, the MWCNT does not fundamentally alter the plant's physiology.

The capacity of the MWCNT to affect the nutrient element delivery to the seedlings was investigated via polarized EDXRF spectrometry. The concentrations of all ionic nutrients were seen to pass through a narrow or 
broadly defined minimum with respect to the MWCNT concentration. We argue that this behavior likely points to the action of two contrary forces: one the CNT ion-transient-dipole (ITD) interaction that tends to restrain entry of the ion/polar species into the plant and the other the water inflow that facilitates entry. The relatively high concentrations of many nutrients generally at $\sim 20 \mathrm{mg} / \mathrm{l}$ of MWCNT tied in with the increase of fresh and dry weights at this MWCNT concentration.

Using pEDXRF spectrometry the interaction of the MWCNT (at $20 \mathrm{mg} / \mathrm{l}$ ) with the +2 and +3 oxidation states of $\mathrm{Fe}$ in relation to the delivery of $\mathrm{Fe}$ as nutrient to the seedling in agarose media was investigated. The results indicated that the ITD interaction between the CNT and the $\mathrm{Fe}$ ions may have a role in the reduction of the $3+$ to the $2+$ oxidation state. $\mathrm{Ca}^{2+}$ concentrations showed a high inverse correlation with $\mathrm{Fe}^{2+}$ suggesting some sort of $\mathrm{Ca}$ efflux from the seedling cells with a possible mechanism being cation exchange in the cell wall matrix. The dry weights and \% water contents generally followed the $\mathrm{Ca}$ elemental concentration trend as would be expected in view of the central role of $\mathrm{Ca}$ in metabolism and cell wall properties.

The surface structure near the black-layer of the pericarp-seed coat in the presence/absence of the MWCNT and $\mathrm{Fe}^{2+} / \mathrm{Fe}^{3+}$, was inspected by SEM. The surface topography showed fairly dense pore-like structures for the pure MWCNT treated case. These pores may account for the enhanced water delivery by the MWCNT. The presence of $\mathrm{Fe}^{2+}$ and $\mathrm{Fe}^{3+}$ ions either without or with the MWCNT showed no such structures. We argue that the adsorption of the Fe ions on the CNT surface may impact its functionality such as its perforation of the seed-coat and its enhancement of germinative growth. The results of the experiment show that the introduction of the $\mathrm{Fe}$ and $\mathrm{Cl}$ ions depreciated the MWCNT's ability to enhance dry weight and to a lesser extent, deliver water to the whole seedling.

We conclude that pristine MWCNTs at low concentrations benefit the growth of maize seedlings by enhancing water, nutrient transport and biomass but that their potency could be diminished by high concentrations of ions/polar species in the medium. These findings suggest a potential for the utilization of CNTs for optimizing water transport in arid-zone agriculture and of improving crop biomass yields.

Acknowledgments DKT thanks CONACyT of Mexico for his doctoral scholarship (No.: 229122). The authors are grateful to Dr. Ariosto Medina Flores for the SEM imaging. NDS thanks Professor Miller B. McDonald of the Horticulture and Crop Science Department, Ohio State University, USA for helpful discussions.

Open Access This article is distributed under the terms of the Creative Commons Attribution License which permits any use, distribution, and reproduction in any medium, provided the original author(s) and the source are credited.

\section{References}

Alexiadis A, Kassinos S (2008) Molecular Simulation of water in carbon nanotubes. Chem Rev 108:5014-5034

Alvarado CJ, Abuhani WA, Whelan T, Castillo OS, Landsberger S, Villaseñor LM, Borjas SE, Bribiesca SL, Alexander SA, Dasgupta-Schubert N (2013) Comparative analysis of the lead and copper concentrations of metal accumulating plants with or without mycorrhizae. Commun Soil Sci Plant Anal (in press)

Begum P, Ikhtiari R, Fugetsu B, Matsuoka M, Akasaka T, Watari F (2012) Phytotoxicity of multi-walled carbon nanotubes assessed by selected plant species in the seedling stage. Appl Surf Sci 262:120-124

Beu TA (2011) Molecular dynamics simulations of ion transport through carbon nanotubes. III. Influence of the nanotube radius, solute concentration and applied electric fields on the transport properties. J Chem Phys 135:044516

Brateman PS, Cairns-Smith AG, Sloper RW, George Truscott T, Craw M (1984) Photo-oxidation of Fe(II) in water between $\mathrm{pH}$ 7.5-4.0. J Chem Soc, Dalton Trans 7:1441-1445

Calabrese EJ, Baldwin LA (2000) Chemical hormesis: its historical foundations as a biological hypothesis. Hum Exp Toxicol 19:2-31

Difco $^{\mathrm{TM}}$ and $\mathrm{BBL}^{\mathrm{TM}}$ manual (2009) 2nd ed, http://www.bd.com/ europe/regulatory/Assets/IFU/Difco_BBL/281230.pdf

Donaldson K, Aitken R, Tran L, Stone V, Duffin R, Forrest J, Alexander A (2006) Carbon Nanotubes: a review of their properties in relation to pulmonary toxicology and workplace safety. Toxicol Sci 92:5-22

Dresselhaus MS, Dresselhaus G, Jorio A (2004) Unusual properties and structure of carbon nano tubes. Annu Rev Mater Res 34:247-278

Enquist BJ (2002) Universal scaling in tree and vascular plant allometry: toward a general quantitative theory linking plant form and function from cells to ecosystems. Tree Physiol 22:1045-1064

Frolov AI, Kirchner K, Kirchner T, Fedorov MV (2012) Molecularscale insights into the mechanisms of ionic liquids' interactions with carbon nanotubes. Faraday Discuss 154:235-247

Heckel J, Haschke M, Brumme M, Schindler R (1992) Principles and applications of energy dispersive $\mathrm{x}$-ray fluorescence analysis with polarized radiation. J Anal At Spectrom 7:281-286

Ijima S (1991) Helical micro-tubules of graphitic carbon. Nature 354:56-58

Khodakovskaya M, Dervishi E, Mahmood M, Xu Y, Li Z, Watanabe F, Biris AS (2009) Carbon nanotubes are able to penetrate plant seed coat and dramatically affect seed germination and plant growth. ACS Nano 3:3221-3227. A Article retracted, ACS Nano, 6, $7541(2012)\}$

Lee BW, Schubert R, Cheung YK, Zannier F, Wei Q, Sacchi D, Sia SK (2010) Strongly binding cell-adhesive polypeptides of programmable valencies. Angew Chem 49:1971-1975

Lin D, Xing B (2007) Phytotoxicity of nanoparticles: inhibition of seed germination and root growth. Environ Pollut 150:243-250

Lin C, Su Y, Takahiro M, Fugetsu B (2010) Multiwalled carbon nanotubes induce oxidative stress and vacuolar structure changes to Arabidopsis T87 suspension cells. Nano Biomed 2:170-181

Liu Q, Chen B, Wang Q, Shi X, Xiao Z, Lin J, Fang X (2009) Carbon nanotubes as molecular transporters for walled plant cells. Nano Lett 9:1007-1010

MacDonald MB (2007) Physiology of seed germination. http:// seedbiology.osu.edu/HCS631_files/4A\%20Seed\%20germination. pdf; http://seedbiology.osu.edu/HCS631_files/4B\%20Seed $\% 20$ germination.pdf

Manara A (2012) Plants and heavy metals. In: Furini A (ed) Springer briefs in biometals. Springer, Berlin 
Martinelli V, Cellot G, Toma FM, Long CS, Caldwell JH, Zentilin L, Giacca M, Turco A, Prato M, Ballerini L, Mestroni L (2012) Carbon nanotubes promote growth and spontaneous electrical activity in cultured cardiac myocytes. ACS Nano Lett 12:1831-1838

McDonald MB, Sullivan J, Lauer MJ (1994) The pathway of water uptake in maize seeds. Seed Sci Technol 22:79-90

Merkoçi A (ed) (2009) Biosensing using nano materials. Wiley, NJ. doi:10.1002/9780470447734

Miskovic ZI (2008) Interactions of ions with carbon nano-structures. J Phys Conf Ser 133:012011. doi:10.1088/1742-6596/133/1/ 012011

Mondal A, Basu R, Das S, Nandy P (2011) Beneficial role of carbon nanotubes on mustard plant growth: an agricultural prospect. J Nanopart Res 13:4519-4528

Panyam J, Labhatsevar V (2003) Biodegradable nanoparticles for gene and drug delivery to cells and tissue. Adv Drug Deliv Rev 55:329-347

Serag MF, Kaji N, Tokeshi M, Baba Y (2012) Introducing carbon nanotubes into living walled plant cells through cellulaseinduced nanoholes. RSC Adv 2:398-400

Srinivasan C, Saraswathi R (2010) Nanoagriculture-carbon nanotubes enhance tomato seed germination and plant growth. Curr Sci 99:274-275

Taiz L, Zeiger E (2006) Plant Physiology, 4th edn. Sinauer Associates Inc, Sunderland
Tiwari A and Tiwari A (eds) (2013) Nano materials in drug delivery, imaging and tissue engineering. Scrivener, Wiley. ISBN: 978-11182-9032-3

Tripathi S, Sonkar SK, Sarkar S (2011) Growth stimulation of gram (Cicer arietinum) plant by water soluble carbon nanotubes. Nanoscale 3:1176

US Environmental Protection Agency (USEPA) (1996) Ecological effects test guidelines (OPPTS 850.4200): seed germination/root elongation toxicity test. Available from: http://www.epa.gov/ opptsfrs/publications/OPPTS_Harmonized/850_Ecological_ Effects_Test_Guidelines/Drafts/850-4200.pdf

Vaisman L, Daniel Wagner H, Marom G (2006) The role of surfactants in dispersion of carbon nanotubes. Adv Colloid Interface Sci 128-130:37-46

Villagarcia H, Dervishi E, Silva K, Biris AS, Khodakovskaya MV (2012) Surface chemistry of carbon nanotubes impacts the growth and expression of water channel protein in tomato plants. Small 8:2328-2334

Wang X, Han H, Liu X, Gu X, Chen K, Lu D (2012) Multi-walled carbon nanotubes can enhance root elongation of wheat (Triticum aestivum) plants. J Nanopart Res 14:841-851

West GB, Brown JH (2005) The origin of allometric scaling laws in biology from genomes to ecosystems: towards a quantitative unifying theory of biological structure and organization. J Exp Biol 208:1575-1592 